EPJ Web of Conferences 57, 02003 (2013)

DOI: $10.1051 /$ epjconf/20135702003

(C) Owned by the authors, published by EDP Sciences, 2013

\title{
Atomic and molecular spectroscopy with optical-frequency-comb-referenced IR coherent sources
}

\author{
P. Cancio ${ }^{1, a}$, S. Bartalini ${ }^{1}$, M. De Rosa ${ }^{2}$, G. Giusfredi ${ }^{1}$, D. Mazzotti ${ }^{1}$, \\ P. Maddaloni ${ }^{2}$, M.S. Vitiello ${ }^{3}$ and P. De Natale ${ }^{4}$ \\ ${ }^{1}$ Istituto Nazionale di Ottica (INO) - CNR and European Laboratory for Non-linear \\ Spectroscopy (LENS), via Carrara 1, 50019 Sesto Fiorentino FI, Italy \\ ${ }^{2}$ Istituto Nazionale di Ottica (INO) - CNR, via Campi Flegrei 34, 80078 Pozzuoli NA, Italy \\ ${ }^{3}$ NEST, Istituto Nanoscienze - CNR and Scuola Normale Superiore, Piazza San Silvestro 12, \\ 56127 Pisa PI, Italy \\ ${ }^{4}$ Istituto Nazionale di Ottica (INO) - CNR, Largo Fermi 6, 50125 Firenze FI, Italy
}

\begin{abstract}
We provide a review of progress in the development of metrological-grade measurements in atomic and molecular systems through the extension, in the mid-infrared and far-infrared range, of optical frequency combs (OFCs) and the introduction of new techniques and highly coherent sources.
\end{abstract}

\section{INTRODUCTION}

The hallmark of progress in physical sciences is the constant improvement of measurement precision and accuracy. Testing ground of such progress are the simplest systems, like atoms and molecules, that can be investigated by spectroscopic techniques. However, their transition frequencies are spread across most part of the electromagnetic spectrum, depending on the specific atom/molecule and degree of freedom. Therefore, their accessibility for experimental studies has always depended on the effective availability of proper sources and techniques. Moreover, accurate measurements require appropriate standards, available for each selected spectral range. Since their first demonstrations at the end of the last century, OFCs have soon become widespread clockworks, providing stable and accurately known frequency references across the visible/near-IR spectral interval [1, 2]. More recently, their extension to other spectral windows has been pursued by many groups, worldwide [3, 4]. Of course, such extensions has always proceeded hand-in-hand with the development of appropriate tunable coherent sources and techniques.

In this paper, we review recent progress in the development of a metrological toolbox enabling precise measurement of atomic and molecular transition frequencies in the infrared range, mainly achieved by groups at the European Laboratory of Nonlinear Spectroscopy (LENS) and National Institute of Optics (INO-CNR) in Florence and Naples, Italy, putting the results in the proper context. More specifically, we will show results for atomic helium transitions around $1083 \mathrm{~nm}$ wavelength [5-10], experiments on $\mathrm{CO}_{2}, \mathrm{CH}_{3} \mathrm{I}$ and $\mathrm{CH}_{4}$ in the 3-4.5 $\mu \mathrm{m}$ range [11-26], novel set-ups for interrogation of rotational transitions in simple molecules in the far infrared (THz) range [27, 28]. As is described, such experiments have been strictly dependent on the development of highly coherent sources, often harnessing their intrinsic properties, and new methodologies.

\footnotetext{
ae-mail: pablo.canciopastor@ino.it
}

This is an Open Access article distributed under the terms of the Creative Commons Attribution License 2.0, which permits unrestricted use, distribution, and reproduction in any medium, provided the original work is properly cited. 

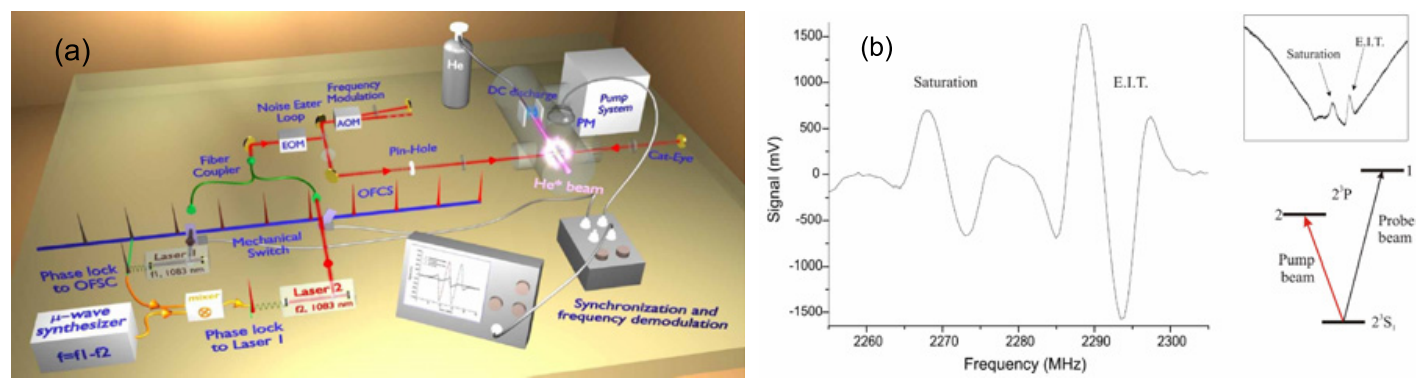

Figure 1. (a) Experimental set-up of the OFC-assisted multi-resonant diode laser spectrometer. (b) EIT spectrum for the $\mathrm{V}$ coherence spectroscopy configuration with $\mathrm{L} 1$ resonant with ${ }^{4} \mathrm{He} 2^{3} \mathrm{~S}_{1} \rightarrow 2^{3} \mathrm{P}_{2}$ transition and L2 resonant with ${ }^{4} \mathrm{He} 2^{3} \mathrm{~S}_{1} \rightarrow 2^{3} \mathrm{P}_{1}$ one, when the $\mathrm{L} 2$ frequency is scanned and L1 frequency is fixed. Saturation dip spectrum due to $\mathrm{L} 2$ is also shown.

\section{HELIUM SPECTROSCOPY WITH AN OFC-ASSISTED MULTI-RESONANT DIODE LASER SPECTROMETER AT 1083 NM}

Helium atom has long been a favorite testing ground for fundamental two-electron QED theory and for new techniques in atomic physics, both experimental and theoretical. In this context, the spectroscopic measurements and theoretical determinations of the energies of low-lying triplet states have pushed the limits of precision. The readily accessible $2^{3} \mathrm{P}$ manifold from the metastable $2^{3} \mathrm{~S}$ state at $1083 \mathrm{~nm}$ has been the focus of much experimental activity, particularly following the introduction of OFC's [5-10, 29]. Fundamental physical constants and atomic properties can be determined for such precise spectroscopic measurements. Fine structure (FS) energies of the $2^{3} \mathrm{P}$ manifold in He can produce an accurate determination of the fine structure constant, $\alpha$ [30]. Nuclear properties, as difference of nuclear charge radius among such different He isotopes, can be determined from precise isotope-shift (IS) measurements at $1083 \mathrm{~nm}$ [9]. Measurements of $2^{3} \mathrm{P}$ hyperfine structure (HFS) of fermionic He isotopes are used to determine nuclear interaction and nuclear magnetic moment in such atomic species [10]. Precise measurements of large energies as optical transitions between $2^{3} \mathrm{~S}$ and $2^{3} \mathrm{P}$ levels are used to determine QED and high-order relativistic effects (Lamb shifts) in such states [5, 6, 9, 29].

Although spectroscopic measurements of such frequency intervals, at least for the microwave FS, HFS and IS energies, can be allowed by heterodyne optical spectroscopy without need of absolute frequency referencing of the laser sources [7, 30, 31], metrological properties transferred by the OFCs are fundamental for accurate measurements of optical frequencies [5, 6, 9, 29]. In addition, FS, HFS and IS microwave intervals, calculated by difference of optical transitions, benefit of such OFC referencing in terms of final accuracy [7, 10]. In this frame, we have recently developed an OFC-assisted diode laser spectrometer at $1083 \mathrm{~nm}$ for multi-resonant spectroscopy of He atom around such wavelength [8] (see Fig. 1a).

Basically two different $1083 \mathrm{~nm}$ Extended Cavity-Distributed Bragg Reflector (EC-DBR) diode laser sources [32], resonant with different He transitions, are phase-locked to a near-IR OFC (MenloSystems, FC1500-100). The first laser (L1) is directly phase-locked to the nearest OFC tooth $N_{1}$, by beating both on a fast photodetector. Phase fluctuations of the CW laser with respect to the comb are corrected by analyzing the detected beatnote, $f_{c 1}$, using a fast optical phase-locked loop correction circuit (OPLL1). For the second laser (L2), we phase-locked it directly to the L1 laser. We followed such approach instead to phase-lock it to the nearest comb tooth as in the case of L1 in order to avoid all excess OFC phase-noise between the two laser frequencies, and hence guaranteeing two lasers with the same spectral characteristics, emitting at different frequencies, but as required by multiplex precision spectroscopy. For frequency separations of tens of $\mathrm{GHz}$, as in the case of He transitions at $1083 \mathrm{~nm}$, directly beating of both $\mathrm{CW}$ lasers on a fast photodetector is enough to determine the phase-correction 
signal for L2. For larger separations, the OFC can be used as an optical frequency mixer to compare and control directly the phase/frequency noise of the two CW lasers, as described in section 3. Finally, a microwave synthesized frequency, $f_{\mu w}$ is used to down convert the detected beat-note, $f_{12}$ to the local oscillator frequency $f_{L O}$ of the second phase-lock circuit (OPLL2). In this way, we have developed a GPS (Global Positioning System) traceable absolute frequency chain for both lasers given by:

$$
\begin{aligned}
& f_{1}=f_{0}+N_{1} f_{r}+f_{L O} \\
& f_{2}=f_{1}+f_{12}=f_{0}+N_{1} f_{r}+f_{\mu w}+f_{L O}
\end{aligned}
$$

where $f_{r}$ and $f_{0}$ are the OFC repetition rate and carrier-envelope offset frequencies controlled against of a Rb-GPS-disciplined 10-MHz quartz clock (stability of $6 \times 10^{13}$ in $1 \mathrm{~s}$ and accuracy of $2 \times 10^{-12}$ ). Moreover, simultaneous synthesized scans of both frequencies are allowed by changing the $f_{r}$ frequency. Alternatively, the frequency difference between the lasers is scanned by changing $f_{\mu w}$. This approach can be extended to a larger number of OFC-assisted laser sources, even in other spectral regions, and with larger frequency separations between them. Such systems are proposed for applications that require phase-locked lasers to a low-power optical reference under noisy conditions.

Sub-Doppler saturation fluorescence spectroscopy on metastable He atomic beam at $1083 \mathrm{~nm}$ was realized with such spectrometer (see Fig. 1b). Simultaneous absolute frequencies of optical transitions and frequency differences between them can be measured at the same level of precision and accuracy. Systematic effects were minimized or avoided, in particular, those due to non resonant light, by means of the alternative blocking of both lasers. Thanks to such precision spectroscopy, we have recently determined the nuclear charge radius of ${ }^{3} \mathrm{He}$ with respect to the ${ }^{4} \mathrm{He}$ one [9], discovering possible unknown effects in the electron-nucleus interaction beyond standard QED. In this case ${ }^{3} \mathrm{He}-{ }^{4} \mathrm{He}$ IS at $1083 \mathrm{~nm}$ was measured by frequency difference of the centroid optical frequencies between $2^{3} \mathrm{~S}$ and $2^{3} \mathrm{P}$ manifolds measured in 2012 for ${ }^{3} \mathrm{He}$ [9] and in 2004 for ${ }^{4} \mathrm{He}$ [5, 6], putting in evidence the potentiality of OFC assisted absolute frequency measurements. These measurements in ${ }^{3} \mathrm{He}$ have allowed by the first time to test the combined FS-HFS QED theory of $2^{3} \mathrm{P}$ manifold in ${ }^{3} \mathrm{He}$ at the $\mathrm{kHz}$ level [10], meaning the first step for a determination of the nuclear magnetic moment of this atom from an atomic physics measurement. Finally, this OFC-assisted multiresonant spectrometer can be used for coherence spectroscopy following Raman schemes, as the one illustrated in Fig. 1b for the Electronically-InducedTransparency (EIT) in ${ }^{4} \mathrm{He}$. Such schemes can be used for applications that require a precise coherent manipulation of individual atomic or molecular quantum states in cold samples [33].

\section{3. $\mathrm{CO}_{2}$ SPECTROSCOPY WITH AN OFC-REFERENCED NON-LINEAR SOURCE AT $4.2-4.5 \mu \mathrm{M}$}

Since 2005, within our research group in Florence, an OFC-referenced difference-frequency-generated (DFG) spectrometer, based on a $\chi^{(2)}$ non-linear process in a periodically-poled $\mathrm{LiNbO}_{3}$ crystal, has being developed and continuously improved, in terms of stability and emitted power $[11,34,35]$. This coherent source is aimed at transferring the clockwork nature (one-step traceability of optical frequencies against the Cs microwave standard at $9.2 \mathrm{GHz}$ ) of OFCs from the visible/near-IR regions to the mid IR, where most of the strongest ro-vibrational molecular transitions lie (the so called "fingerprint region", between 2.5 and $10 \mu \mathrm{m})$.

In our earlier experiment [11] we used the OFC only as an absolute frequency reference for our DFG source. By coupling this mid-IR radiation to a medium-finesse $(F \sim 500)$ cavity, we performed saturated-absorption sub-Doppler spectroscopy and we measured the line-center absolute frequency of the $\left(00^{0} 1-00^{0} 0\right) \mathrm{R}(60)$ transition of $\mathrm{CO}_{2}$ at $2384.994 \mathrm{~cm}^{-1}$, with an accuracy of about $10 \mathrm{kHz}$. We also performed a preliminary cavity-ring-down $(\mathrm{CRD})$ experiment, with low $(<1 \%)$ coupling efficiency, due to a radiation linewidth much larger $(<1 \mathrm{MHz})$ than the high-finesse cavity mode $(<10 \mathrm{kHz})[12]$. 


\section{EPJ Web of Conferences}

More recently the locking scheme was changed, in order to use the OFC also as a transfer oscillator for phase-locking the pump radiation involved in the DFG process (delivered from an external-cavity diode laser) to the signal one (delivered from a fiber-amplified Nd : YAG laser). The phase-locking technique is based on a scheme involving a direct-digital-synthesis process able to cancel out any phase-noise contribution from the two free parameters of the OFC: the carrier-envelope offset $f_{0}$ and the repetition frequency $f_{r}$ [36]. In this way, the extremely high frequency stability of the latter source (based on a monolithic non-planar ring oscillator laser cavity) is directly transferred to idler radiation. The achieved intrinsic linewidth of the mid-IR source was measured to be as low as $10 \mathrm{~Hz}$ [34]. Such a narrow emission spectrum of the source has turned to be crucial for efficiently coupling radiation to a high-finesse cavity with a few $\mathrm{kHz}$ mode linewidth.

Two years ago, we developed a high-sensitivity technique for trace gas sensing, named saturatedabsorption cavity ring-down (SCAR), in order to overcome the main limitation to the achievable CRD sensitivity, namely variations of the empty-cavity decay rate [13]. The SCAR working principle can be shortly described as follows. An optical cavity filled with an absorbing molecular species is illuminated with an intense CW laser tuned to excite the target molecular transition of the gas. When the laser is turned off, photons stored in the cavity decay due to both absorption from the gas and mirror leakage. Because light intensity saturates the molecular transition, the initial instants of decay are affected by losses from the mirrors only. Once this background is subtracted, within each CRD event, we can determine the absolute quantity of the trace gas species from the linear molecular absorption encoded in the decay tail. In the same work, we demonstrated both the high sensitivity and the high spectral resolution of SCAR, by measuring the hyperfine structure of the ${ }^{17} \mathrm{O}^{12} \mathrm{C}^{16} \mathrm{O}$ isotopologue in natural abundance.

In case of extremely low concentrations of the absorbing molecular species, the SCAR cavity must be filled at higher gas pressures, in order to increase the absorption coefficient to be measured. Higher pressures require higher intensity to saturate the transition and thus higher power from the mid-IR radiation source. This was achieved by placing the non-linear crystal inside a Ti : sapphire laser cavity, boosting the DFG power up to $30 \mathrm{~mW}$ [35].

The most prominent result obtained by the combination of the SCAR technique with the powerboosted DFG source was the first optical detection of ${ }^{14} \mathrm{C}^{16} \mathrm{O}_{2}$ well below the $\sim 1$-ppt natural abundance [15]. After a systematic spectroscopic measurement of the $\left(00^{0} 1-00^{0} 0\right)$ ro-vibrational band of this elusive isotopologue, by using an enriched sample, we improved the ground-state molecular constants of ${ }^{14} \mathrm{C}^{16} \mathrm{O}_{2}$ by one order of magnitude [14]. We also identified the $\mathrm{P}(20)$ transition as the target line for radiocarbon detection, being the best trade-off between the highest linestrength and the lowest interference from nearby lines of more abundant $\mathrm{CO}_{2}$ isotopologues. The intercomparison between concentrations measured by SCAR and by the accelerated mass spectrometry (AMS) was successfully checked, finding a very good agreement within respective uncertainties. At last, the almost perfect linearity of SCAR was confirmed also at high enrichment levels (up to 6400 times the natural abundance) [16].

\section{4. $\mathrm{CO}_{2}$ SPECTROSCOPY WITH AN OFC-REFERENCED QCL SOURCE AT $4.3 \mu \mathrm{M}$}

Quantum Cascade Lasers (QCLs) represent the extension of the semiconductor lasers family to the mid-IR region, where conventional diode lasers can not operate due to fundamental limitations of their physics. Their features make them the ideal sources for a variety of mid-IR applications in the "real-word", where compact size, low electrical power consumption together with high optical output power are key features for practical and industry-oriented use. Beside this, QCLs have also shown remarkable performances from the point of view of their spectral purity: the short lifetime of the upper lasing level, due to the presence of efficient non-radiative channels, produces a positive benefit on the intrinsic linewidth, which is of the order of hundreds $\mathrm{Hz}$ [37]. This means that QCLs can be used as very monochromatic sources, once provided an efficient technique for stabilizing their frequency. In 


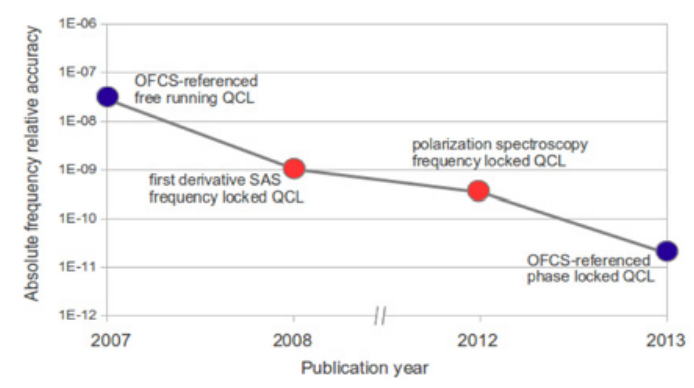

Figure 2. Improvement of the relative accuracy of the absolute frequency measurements made by QCL-based spectroscopic experiments, in the last years. The four points are related to our four works cited in this section: Refs. [17-20], respectively.

fact, in typical operating conditions, they are affected by a larger frequency noise, that broadens their spectrum up to few $\mathrm{MHz}$, which arises partly from the current noise of the driving current and partly from the active region itself, for some still unexplained mechanisms [38]. In this framework, a crucial step towards an extensive use of QCLs for demanding spectroscopic and metrological applications is the development of techniques enabling the narrowing of the QCL emission and its referencing to a stable frequency standard. In last years we developed several techniques for either giving an absolute frequency reference to a mid-IR QCL or for further narrowing its emission linewidth. They can be divided in two different categories: those directly based on an OFC, and those based on a secondary frequency standard, such as a molecular transition. In the following we will briefly describe experiments from both these categories, demonstrating how the accuracy of the spectroscopic measurement on $\mathrm{CO}_{2}$ molecular transitions has been progressively improved by more than 3 orders of magnitude (Fig. 2). Starting from the second category, it is worth mentioning the first stabilization of a mid-IR QCL to a sub-Doppler feature of $\mathrm{C} \mathrm{CO}_{2}$ line, obtained by using the first derivative of a saturated-absorption Lamb dip for closing a feed-back loop acting on the QCL current [18]. In this case we achieved the stabilization of the QCL absolute frequency with an overall accuracy of $10^{-9}$ but, due to the poor bandwidth of the lock-in based detection, we did not achieve any narrowing of the QCL source. This last result was indeed obtained by changing the spectroscopic sub-Doppler technique: adopting a polarization spectroscopy set-up we have recently obtained the narrowing of the emission linewidth of a QCL from its typical free-running value of about $1 \mathrm{MHz}$ (FWHM) down to less than $1 \mathrm{kHz}$ [19]. As a consequence, also the stabilization of the QCL frequency at the center of the molecular transition was improved, achieving an overall accuracy on the QCL absolute frequency of about $4 \times 10^{-10}$, mainly limited by systematic errors. The techniques described above, as already noted, do not require an OFCS, and thus are very compact and cost effective solutions. On the other hand, since they are based on the stabilization of the frequency to a fixed point, they do not allow to tune the QCL frequency. For this reason we also worked on the referencing of a mid-IR QCL to an OFC. To this purpose, a frequency conversion is required, since the OFC operates in the near-IR range. We both demonstrated an approach based on the up-conversion of the QCL frequency to the near-IR region, where the beating with the OFCS is observed [17], and an approach based on the observation of the beat-note directly in the mid-IR, by beating the QCL with the ultra-narrow DFG source described in the previous section. In the first case the absolute frequency of a free-running QCL was counted while the QCL was scanning across a Doppler-broadened molecular transition; the relative accuracy of the frequency determination was evidently limited by the QCL emission linewidth, at about $3 \times 10^{-8}$. For this reason, in the second case, a phase lock of the QCL to the OFC-referenced DFG source was implemented. In this way, not only the possibility of continuously tuning the QCL frequency is preserved, but also a dramatic narrowing of the QCL emission is achieved. With this scheme, the absolute QCL frequency is known with an accuracy of about $10^{-12}$, while the center of a $\mathrm{CO}_{2}$ molecular line has been measured with an accuracy of about $2 \times 10^{-11}$ [20]. 
(a)

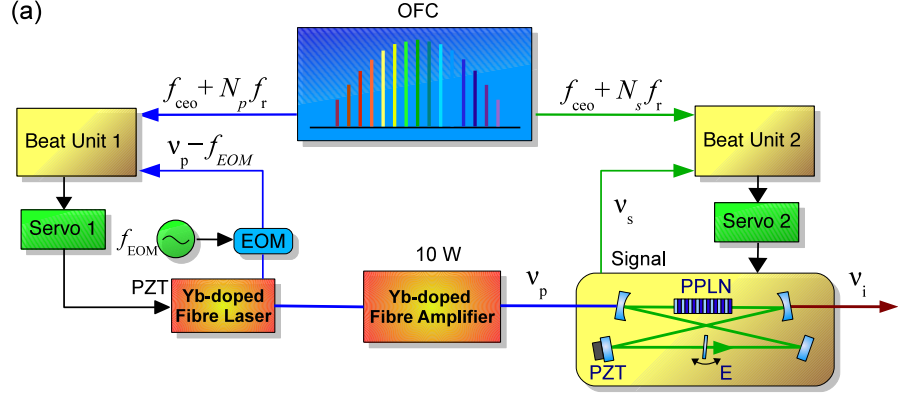

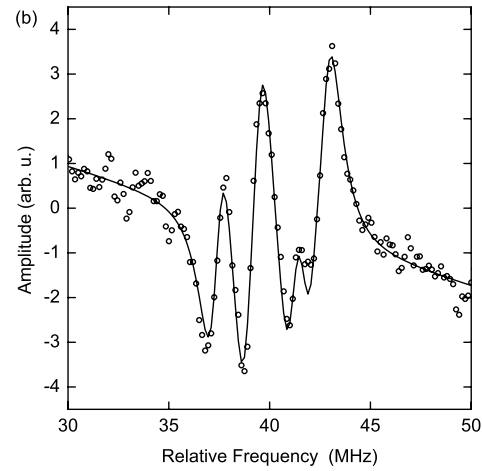

Figure 3. (a) Simplified scheme of the OPO locking to the optical frequency comb (OFC). PPLN: periodicallypoled lithium niobate crystal; E: YAG etalon; EOM: electro-optic modulator. (b) Hyperfine structure of the $v_{1}$ $\mathrm{P}(18,3)$ rovibrational transition of $\mathrm{CH}_{3} \mathrm{I}$, at 30 mTorr, with $630 \mathrm{~mW}$ of saturating power. Experimental data (open circles) have been fitted by a linear background and six Lorentzian derivatives.

\section{5. $\mathrm{CH}_{3}$ I SPECTROSCOPY WITH AN OFC-REFERENCED OPO SOURCE AROUND $3 \mu \mathrm{M}$}

For many years since their first demonstration CW optical parametric oscillators (OPOs) have been considered impracticable as spectroscopic source, as their operation was affected by strong instabilities or high pump power were needed for exceed the oscillation threshold. In the last decade, advances in material science and the availability of powerful and narrow-linewidth laser sources in the near IR renewed the interest in OPOs. In the last years several CW OPOs have been demonstrated as reliable source for high-resolution spectroscopy. They are mostly addressed to the generation of coherent radiation in the mid-IR region between 2 and $5 \mu \mathrm{m}$, a region of particular interest, because of the presence of strong fundamental rovibrational bands of many molecules. OPOs can generate new frequencies over a wide spectral range from visible to IR, with emitted power up to watt level and with good spectral features.

We developed a widely-tunable, singly-resonant OPO, emitting more than $1 \mathrm{~W}$ in the region between 2.7 and $4.2 \mu \mathrm{m}$. The OPO performance has been demonstrated by carrying out high resolution spectroscopic measurements on rovibrational transitions of $\mathrm{CH}_{3} \mathrm{I}$ vapor, being able to resolve their subDoppler hyperfine structure and determine their absolute frequencies.

The OPO is based on a 50-mm-long periodically-poled sample of $\mathrm{MgO}: \mathrm{LiNbO}_{3}$, with seven different grating periods. The crystal is placed in a four mirror ring cavity, resonating at the signal wavelengths $(1410-1800 \mathrm{~nm})$. An amplified, narrow linewidth $(40 \mathrm{kHz}$ at $1 \mathrm{~ms}) \mathrm{Yb}$-doped fibre laser is used as pump for the OPO. Thus, by properly choosing the crystal temperature and the grating period, the idler frequency in the mid IR can be continuously tuned in the range between 2.7 and $4.2 \mu \mathrm{m}$, with typical emitted power of $\sim 1 \mathrm{~W}$ [22]. Fine frequency tuning can be accomplished both by changing the pump laser and the signal frequencies. Stable and accurate operation can be achieved by locking both the signal and pump frequencies to a self-referenced OFC [21]. Indeed, the octave-spanning comb (between 1 and $2 \mu \mathrm{m}$ ), generated by a mode-locked Er-doped fibre laser, includes both the pump and the signal frequencies of the OPO. Repetition rate and offset frequency of the comb are stabilized against a 10MHz BVA quartz oscillator, which is disciplined by to a Rb-GPS clock. The comb radiation is coupled into two optical fibers and combined with few milliwatts of pump and signal light, respectively (Fig. 3). An InGaAs fast photodiode is used to detect the beat note $f_{\mathrm{b} 1}\left(f_{\mathrm{b} 2}\right)$ of the pump (signal) and the nearest comb tooth. After bandpass filtering and amplification each beat note is mixed with a local oscillator at frequency $f_{\mathrm{LO}}=12 \mathrm{MHz}$, and the IF output of the mixer is sent to the servo electronics. Frequency scan of a few tens of $\mathrm{MHz}$ can be accomplished by offset-locking the pump laser to the comb. For this purpose, a small fraction of the laser beam is deviated towards an electro-optic modulator, which adds 
two sidebands at frequencies $\pm f_{\mathrm{EOM}}$ with respect to the carrier laser frequency $v_{\mathrm{p}}$. In fact, a sideband is locked to the comb, while the OPO is pumped at the carrier frequency. By changing $f_{\mathrm{EOM}}$ the pump frequency and, as a result, the idler frequency are scanned. When both the signal frequency and the pump sideband are simultaneously locked their frequencies can be written as $v_{p}=f_{0}+N_{p} f_{\mathrm{r}}+f_{\mathrm{b} 1}+f_{\mathrm{EOM}}$ and $v_{s}=f_{0}+N_{s} f_{\mathrm{r}}+f_{\mathrm{b} 2}$, respectively. $N_{p}\left(N_{s}\right)$ is an integer number that indexes the pump (signal) nearest comb tooth. The resulting idler frequency reads as

$$
v_{i}=v_{p}-v_{s}=\left(N_{p}-N_{s}\right) f_{\mathrm{r}}+f_{\mathrm{b} 1}-f_{\mathrm{b} 2}+f_{\mathrm{EOM}},
$$

independent of the comb offset frequency $f_{0}$. The locked system showed a relative Allan deviation of $\lesssim 3 \times 10^{-12} \tau^{-1 / 2}$, for $\tau$ between 1 and 200 s [21].

In order to test the spectral features of the absolutely stabilized OPO we carried out sub-Doppler spectroscopy of $\mathrm{CH}_{3} \mathrm{I}$ rovibrational transitions around $3.38 \mu \mathrm{m}$. Rovibrational levels of this molecule, identified by the quantum numbers $J$ and $K$, are split into hyperfine sublevels by electric quadrupole interaction of the iodine nucleus, with nuclear spin $I=5 / 2$. These hyperfine sublevels give rise to six transitions satisfying the selection rule $\Delta F=\Delta J$, where $\mathbf{F}=\mathbf{J}+\mathbf{I}$ [39].

Figure (3b) shows the six sub-Doppler hyperfine components of the $v_{1} \mathrm{P}(18,3)$ rovibrational transition of $\mathrm{CH}_{3} \mathrm{I}$, at $30 \mathrm{mTorr}$ pressure. Phase-sensitive lock-in detection has been adopted to increase the signal-to-noise ratio of the saturated absorption. The profile has been fitted to a linear background and six Lorentzian profile derivatives with equal widths and amplitudes. The centroid of the multiplet has been estimated as $88791204.19 \pm 0.05 \mathrm{MHz}$. The $50 \mathrm{kHz}$ uncertainty is the standard deviation of the fit results from different data sets. The position of the six components with respect to the centroid are in good agreement with the calculated ones. Also, we estimated an upper limit of $200 \mathrm{kHz}$ for the idler linewidth (FWHM).

In a singly resonant OPO the idler linewidth depends on the pump and the signal linewidths. In order to further reduce the idler linewidth, the signal frequency has been stabilized with respect to a resonance of a reference Fabry-Pérot cavity, by means of the Pound-Drever-Hall (PDH) locking scheme [40]. The reference cavity is made by two curved mirrors $(\mathrm{ROC}=1 \mathrm{~m})$ glued on an invar spacer. The mirrors provide high finesse $(F>4000)$ over the same wavelength range of the OPO mirrors. The invar spacer is held by a mechanical suspension and placed in a thermally stabilized vacuum chamber for seismic and acoustic isolation. A servo electronics is fed by the PDH signal and drives the piezo-mounted mirror of the OPO cavity. With a servo-loop bandwidth of about $4 \mathrm{kHz}$, limited by the piezo resonances, we calculated, from the in-loop power spectral density [41], a FWHM of $\sim 70 \mathrm{kHz}$ at $1 \mathrm{~ms}$ for the signal frequency [22]. This linewidth is, to the best of our knowledge, the lowest value reported for a mid IR OPO over $1 \mathrm{~ms}$. In fact, for longer times the reference cavity shows a long term drift of $\sim 1 \mathrm{MHz} / \mathrm{s}$ which is transferred to the locked signal frequency.

\section{6. $\mathrm{CH}_{4}$ SPECTROSCOPY WITH AN OFC-REFERENCED DFG SOURCE AROUND $3 \mu \mathrm{M}$}

With its isotopologues, the $\mathrm{CH}_{4}$ molecule represents the simplest stable hydrocarbon species. For this reason, precision spectroscopy of methane plays a crucial role both in fundamental studies and in many diverse applications. Just to mention a few, these include astrophysical research (methane is an important atmospheric constituent of planets, brown dwarfs, "cold" stars, etc.), absolute frequency metrology, environmental applications (indeed, after water and carbon dioxide, $\mathrm{CH}_{4}$ is the third major greenhouse gas in the Earth's atmosphere), and combustion processes. In particular, the strong $v_{3}$ (stretching) fundamental band (at $3019 \mathrm{~cm}^{-1}$ ) has always been the object of accurate investigations and, in this regard, among all the recently developed IR laser sources, DFGs in periodically-poled non-linear crystals have proved to be the most reliable ones for high-resolution spectroscopy [42]. Figure 4 shows the DFG spectrometer implemented by the Naples group [43]. Here, coherent radiation tunable between 2.9 and $3.5 \mu \mathrm{m}$ is produced starting from a $1 \mu \mathrm{m}$ pump and a $1.5 \mu \mathrm{m}$ signal beam. The latter, tunable between 1540 and $1600 \mathrm{~nm}$, originates from an external-cavity diode laser (ECDL) 


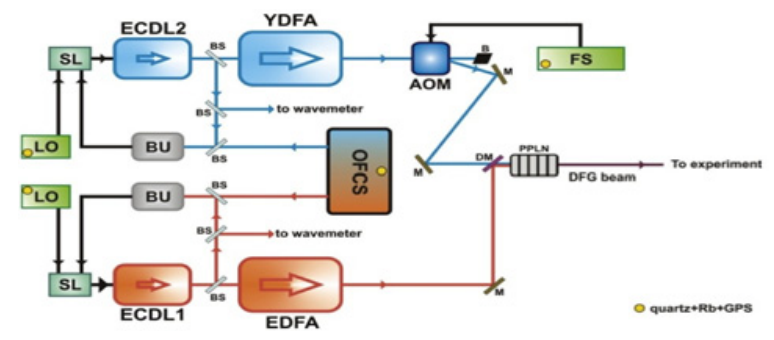

Figure 4. Layout of the experimental apparatus: the OFC-referenced DFG radiation is used for precision spectroscopy. The following legend holds: OFCS $=\mathrm{OFC}, \mathrm{ECDL}=$ external-cavity diode laser, EDFA $=$ Er-doped fiber amplifier, YDFA = Yb-doped fiber amplifier, $\mathrm{SL}=$ servo lock, $\mathrm{LO}=$ local oscillator, $\mathrm{BU}=$ beat unit, $\mathrm{BS}=$ beam splitter, $\mathrm{M}=$ mirror, $\mathrm{DM}=$ dichroic mirror, $\mathrm{FS}=$ frequency synthesizer, $\mathrm{PPLN}=$ periodically poled lithium niobate.

power-boosted up to $10 \mathrm{~W}$ by an Er-doped fiber amplifier (EDFA). The pump radiation, tunable between 1030 and $1070 \mathrm{~nm}$, comes from a second ECDL which seeds an Yb-doped fiber amplifier (YDFA) whose output beam (about $1 \mathrm{~W}$ ) passes through an acousto-optic modulator (AOM). The emerging first-order diffracted beam is superimposed with the signal radiation and then focused into a 5-cmlong, temperature-controlled, multiple-channel periodically poled $\mathrm{LiNbO}_{3}$ (PPLN) crystal, where the DFG process takes place. The resulting idler beam, filtered from the unconverted near-IR radiation by a Ge window, has a power of a few milliwatts and an emission linewidth of approximately $1 \mathrm{MHz}$ (basically determined by the linewidths of the pumping sources). Its absolute frequency is determined by referencing both the ECDLs to a near IR OFC covering the 1-2 $\mu$ m octave (MenloSystems, FC1500$250)$. For this purpose, each beat note at frequency $f_{(s, \text { beat })}\left(f_{(p, \text { beat })}\right)$ between signal (pump) and the $N_{s}\left(N_{p}\right)$ comb tooth is sent to a corresponding electronic servo. The two servo circuits lock these beat notes to given local oscillator values by feeding back proper corrections both to the external-cavity piezos and to the laser current drivers. In this way, the DFG emission frequency reads

$$
f_{D F G}=M f_{r}+\left(f_{(p, \text { beat })}-f_{(s, \text { beat })}\right)+f_{A O M}
$$

where $M=N_{p}-N_{S}, f_{r} \sim 250 \mathrm{MHz}$ is the comb mode spacing and $f_{A O M} \sim 90 \mathrm{MHz}$ is the frequency of the signal driving the AOM. The link to the Cs-clock standard is established by stabilizing $f_{r}$ against a high-quality 10-MHz BVA quartz oscillator which is disciplined, in turn, by a Rb/GPS clock; the same reference chain is used to lock the time base of the frequency synthesizers generating the signals at $f_{(p, \text { beat })}, f_{(s, \text { beat })}$, and $f_{A O M}$, respectively. Finally, the integers $N_{p}$ and $N_{s}$, and hence $M$, are determined by means of a near-IR wavemeter having an accuracy much better than $f_{r} / 2$. In this way, the absolute frequency of the DFG radiation can be monitored by simultaneously measuring the terms of Eq. (3). Coarse tuning of DFG across the molecular resonance is accomplished by changing $M$, while fine steps are realized by slightly changing $f_{r}$ at a given $M$. Originally developed to perform saturation subDoppler spectroscopy of methane [23], such a versatile radiation source has then been used in a number of other detection schemes including

- cavity ring-down spectroscopy (CRDS);

- frequency modulation (FM) spectroscopy [24]; in this frame, a portable DFG-based spectrometer was also realized for detection of methane in natural abundance. Here, the IR beam was coupled to a 13-m multiple-reflection cell and a two-tone FM technique was implemented, thus leading to a minimum detectable concentration of $400 \mathrm{ppt} \mathrm{Hz}^{-1 / 2}$;

- off-axis integrated cavity output spectroscopy (OA-ICOS) [25, 26]. In this experiment, a minimum detectable absorption coefficient of $5.7 \times 10 \mathrm{~cm}^{-1} \mathrm{~Hz}^{-1 / 2}$ was obtained, comparable with other cavity enhanced spectroscopic techniques. 

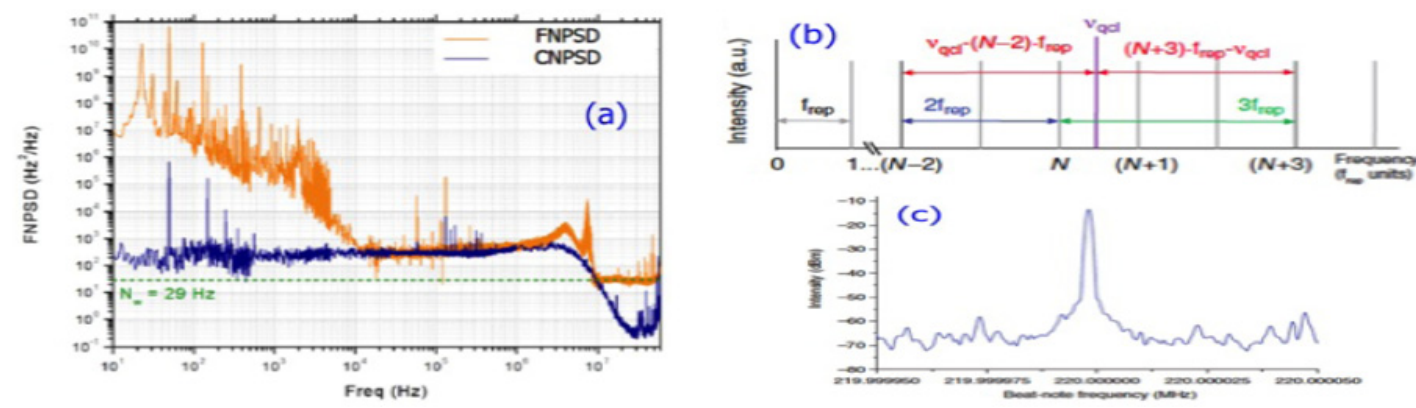

Figure 5. a) Experimental FNPSD of the THz QCL (orange trace), compared with the contribution to the frequencynoise of the CNPSD from the current driver (blue trace). The dashed line marks the white-noise level; (b) Comb-like structure of the THz-FC beam, in the frequency domain, and monochromatic QCL emission $\left(v_{q c l}\right)$. The red-coloured arrows indicate the beating between the QCL and the third-order neighbors teeth of the comb. The corresponding frequencies fall in the range between $2 f_{\text {rep }}$ and $3 f_{\text {rep }}$ (blue and green arrows, respectively); (c) Phase-locked beatnote signal acquired with a $100 \mathrm{~Hz}$ span and $1 \mathrm{~Hz}$ RBW.

\section{MOLECULAR SPECTROSCOPY WITH THZ QCL CONTROLLED WITH A FREE-SPACE THZ FREQUENCY COMB}

Although QCL is the most efficient compact semiconductor source in the mid-IR (4.3-24 $\mu \mathrm{m}), \mathrm{QCL}$ technology is however not still completely mature in the spectral region $>100 \mu \mathrm{m}$. After the first demonstration in 2002 [44], efficient and miniaturized QC sources operating in the $1.2-4.9 \mathrm{THz}$ window have now been successfully developed, in either single-plasmon or double-metal waveguide configuration. Frequency and phase stabilized, high power and reliable, metrological-grade solidstate terahertz sources can indeed find application in a large number of fields, spanning from far-IR astronomy [45] and high-precision molecular gas spectroscopy [46, 47] to high-resolution coherent imaging and telecommunications $[48,49]$. To address such application requirements, high-frequencystability sources are almost mandatory. In this context, the knowledge of the intrinsic linewidth (LW) due to quantum noise plays a key role, ultimately determining the achievable spectral resolution and the coherence length. In a QCL, environmental effects such as temperature, bias-current fluctuations or mechanical oscillations are expected to significantly affect the LWs. We recently developed a sophisticated experimental technique to investigate the spectral purity of a $2.5 \mathrm{THz} \mathrm{QCL}$ via the measurement of its frequency-noise power spectral density (FNPSD). The latter provides, for each frequency, the amount of noise contributing to the spectral width of the laser emission by deriving the laser emission spectrum over any accessible time scale. It also allows calculating the LW reduction achievable using a frequency-locking loop, once its gain/bandwidth characteristics are known [27, 37]. Intensity measurements were performed to retrieve information in the frequency domain by converting the laser frequency fluctuations into detectable intensity (amplitude) variations [37]. As a discriminator, we used the side of a Doppler-broadened methanol molecular transition. Specifically, the ro-vibrational molecular transition line of $\mathrm{CH}_{3} \mathrm{OH}$, centered at $v_{0}=2.5227816 \mathrm{THz}$, was used as a discriminator. The resulting FNPSD spectrum is plotted in Fig. 5a, together with the current-noise power spectral density (CNPSD) of the current driver, converted to the same units by using the current tuning coefficient. Above $8 \mathrm{MHz}$, an asymptotic flattening is observed in the FNPSD, with a significant deviation from CNPSD, thus suggesting a flattening to a white noise level $(N w)$, from which an intrinsic LW $\delta v=\pi$ $\mathrm{Nw}=90 \pm 30 \mathrm{~Hz}$ has been extracted [27]. Despite the broadening induced by thermal photons, the measured LW results narrower than in any other semiconductor laser.

Such an ultra-narrow source has been then employed to demonstrate a free-space terahertz comb as a mature metrological tool. The $2.5 \mathrm{THz}$ QCL has been directly beaten and phase-locked 
using a commercial hot-electron bolometer (HEB) detector, with only a few tens of nW radiation power required. A THz-FC covering the $0.1-6 \mathrm{THz}$ frequency range, has been realized by an optical rectification approach, based on the Cherenkov effect of a femtosecond mode-locked $\mathrm{Ti}$ : sapphire laser in a waveguide fabricated on a $\mathrm{MgO}$-doped $\mathrm{LiNbO}_{3}$ crystal plate [50]. The beating between the QCL and the THz-FC is detected on a HEB (see Fig. 5b). The two beams are superimposed by means of a highly asymmetrical beam splitter, so that more than $99.5 \%$ of the QCL radiation is available for the experiment beam. The QCL terahertz radiation also works as local heater for the superconductive junction, bringing it at the suitable operating temperature. The beat note is mixed with a synthesized RF signal, so that it is down-converted to the $21.4 \mathrm{MHz}$ frequency of the phase-lock electronics local oscillator (LO). The output of the phase detector is processed by a standard proportional-integral-derivative (PID) unit and then sent to the QCL current driver, to close the locking loop. The achieved signal-to-noise ratio is $>50 \mathrm{~dB}$ at $1 \mathrm{~Hz} \mathrm{RBW}$, close to the expected limit of $60 \mathrm{~dB}$ [28]. By numerical integration of the beat-note spectra, we find that about $75 \%$ of the QCL power is phase-locked to the THz-FC emission. The phaselock leads to a narrowing of most of the CW laser emission down to the terahertz comb tooth linewidth (see Fig. 5c). Our approach demonstrates that even a small fraction $(<100 \mathrm{nW})$ of the radiation emitted from the QCL is sufficient to generate a beat note suitable for phase-locking to the comb, paving the way to novel metrological-grade terahertz applications, including high-resolution spectroscopy, manipulation of cold molecules, astronomy and telecommunications [28].

\section{References}

[1] J. Reichert, et al., Phys. Rev. Lett. 3232 (2000).

[2] D. J. Jones, et al., Science 288, 635 (2000).

[3] T. Udem, R. Holzwarth, T. W. Hansch, Nature 416, 233240 (2002).

[4] P. Maddaloni, P. Cancio Pastor, P. De Natale, Meas. Sci. Technol. 20, 052001 (2009).

[5] P. Cancio Pastor, et al., Phys. Rev. Lett. 92, 023001 (2004).

[6] P. Cancio Pastor, et al., Phys. Rev. Lett. 97, 139903 (2006).

[7] G. Giusfredi, et al., Can. J. Phys. 83, 301 (2005).

[8] L. Consolino, et al., Opt. Express 19, 3155 (2011).

[9] P. Cancio Pastor, et al., Phys. Rev. Lett. 108, 143001 (2012).

[10] K. Pachucki, V. Yerokhin, P. Cancio Pastor, Phys. Rev. A 85, 042517 (2012).

[11] D. Mazzotti, et al., Opt. Lett. 30, 997 (2005).

[12] D. Mazzotti, et al., J. Opt. A 8, S490 (2006).

[13] G. Giusfredi, et al., Phys. Rev. Lett. 104, 110801 (2010).

[14] I. Galli, et al., Mol. Phys. 109, 2267 (2011).

[15] I. Galli, et al., Phys. Rev. Lett. 107, 270802 (2011).

[16] I. Galli, et al., Radiocarbon (2012), submitted.

[17] S. Bartalini, et al., Opt. Lett. 32, 988 (2007).

[18] S. Borri, et al., Opt. Express 16, 11637 (2008).

[19] F. Cappelli, et al.. Opt. Lett. 37 (2012).

[20] I. Galli, et al., Appl. Phys. Lett. (2012), to be submitted.

[21] I. Ricciardi, et al., Opt. Express 20, 9178 (2012).

[22] I. Ricciardi, et al., Mol. Phys. 110, 2103 (2012).

[23] P. Maddaloni, P. Malara, G. Gagliardi, P. De Natale, Appl. Phys. B 80, 141 (2005).

[24] P. Maddaloni, P. Malara, G. Gagliardi, P. De Natale, Appl. Phys. B 85, 219 (2006).

[25] P. Malara, P. Maddaloni, G. Gagliardi, P. De Natale, Opt. Express 14, 1304 (2006).

[26] P. Maddaloni, P. Malara, G. Gagliardi, P. De Natale, J. Opt. Soc. Am. B 23, 1938 (2006).

[27] M. Vitiello, et al., Nature Photon. 6, 525 (2012).

[28] L. Consolino, et al., Nature Commun. 3, 1040 (2012).

[29] R. van Rooij, et al., Science 333, 196 (2011). 
[30] M. Smiciklas, D. Shiner, Phys. Rev. Lett. 104, 070403 (2010).

[31] F. Minardi, et al., Phys. Rev. Lett. 82, 1112 (1999).

[32] M. Prevedelli, et al., Opt. Commun. 125, 231 (1996).

[33] W. Vassen, et al., Rev. Mod. Phys. 84, 175 (2012).

[34] I. Galli, et al., Opt. Express 17, 9582 (2009).

[35] I. Galli, et al., Opt. Lett. 35, 3616 (2010).

[36] H. R. Telle, B. Lipphardt, J. Stenger, Appl. Phys. B 74, 1 (2002).

[37] S. Bartalini, et al., Phys. Rev. Lett. 104, 083904 (2010).

[38] S. Borri, et al., IEEE J. Quam. Electron. 47, 984 (2011).

[39] C. H. Townes, A. L. Schawlow, Microwave Spectroscopy (Dover, New York, 1975).

[40] R. Drever, et al., Appl. Phys. B 31, 97 (1983).

[41] G. Di Domenico, S. Schilt, P. Thomann, Appl. Opt. 49, 4801 (2010).

[42] S. Okubo, et al., Opt. Express 19, 23878 (2011).

[43] P. Maddaloni, et al., J. Chem. Phys. 133, 154317 (2010).

[44] R. Köhler, et al., Nature 417, 156 (2002).

[45] J. M. Reix, et al., Acta Astron. 34, 130 (2009).

[46] D. M. Mittleman, Sensing with THz Radiation (Springer, 2003).

[47] P. De Natale, et al., Appl. Opt. 36, 8526 (1997).

[48] M. Tonouchi, Nature Photon. 1, 97 (2007).

[49] F. Capasso, et al., IEEE J. Quantum Electron. 38, 511 (2002).

[50] S. B. Bodrov, et al., Opt. Express 17, 1871 (2009). 